\title{
Which patients should be treated
}

\section{with anti-IgE?}

\author{
K-M. Beeh
}

\begin{abstract}
In the European Union, omalizumab is indicated as add-on therapy to improve asthma control in adult and adolescent patients ( $\geqslant 12 \mathrm{yrs}$ of age) with severe persistent allergic asthma who have a positive skin test or in vitro reactivity to a perennial aeroallergen, and who, despite receiving daily high-dose inhaled corticosteroids and a long-acting $\beta_{2}$-agonist, have the following characteristics: reduced lung function (forced expiratory volume in one second $<80 \%$ ); frequent daytime symptoms or night-time awakenings; and multiple documented severe asthma exacerbations. Omalizumab is indicated for patients with baseline total immunoglobulin E levels of 30-700 IU $\cdot \mathrm{mL}^{-1}$. Patients who receive omalizumab should be assessed by their physician after 16 weeks and treatment continued only if there has been a marked improvement in asthma control. Omalizumab is administered by subcutaneous injection every 2 or 4 weeks at a dosage determined using a dosing table based on the patient's pre-treatment serum total immunoglobulin E levels and body weight.
\end{abstract}

KEYWORDS: Clinical efficacy, exacerbations, omalizumab, patient selection, severe asthma

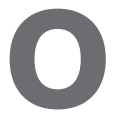
malizumab has shown significant benefits in patients with severe persistent allergic asthma who remain symptomatic despite treatment with high-dose inhaled corticosteroids (ICS) in combination with a longacting $\beta_{2}$-agonist (LABA) [1]. However, careful selection of patients for omalizumab treatment is required to maximise its cost effectiveness. In general, patients will have a high individual burden of disease and clear unmet clinical need, and will include those who are identified as highrisk for asthma-related mortality [2], have $\geqslant 2$ severe exacerbations per year $[1,2]$, require multiple courses of oral corticosteroids per year [3], have regular disruptive nocturnal or daytime symptoms [4] or have impaired quality of life as a consequence of asthma symptoms and exacerbations [4].

In patients with severe persistent asthma, the Global Initiative for Asthma (GINA) 2005 guidelines recommend the use of high-dose ICS and a LABA, with one or more add-on controller medications if required (step 4 therapy), to maintain control of asthma symptoms. The GINA 2005 guidelines include omalizumab as a treatment step 4 add-on therapy [4], and it is the only step 4 add-on therapy with Category A

\footnotetext{
${ }^{1}$ See Post-meeting note on page 88.

This is an updated version of the article owing to an error in Figure 1 in the previous version.
}

evidence (rich body of evidence from randomised controlled trials).

\section{EUROPEAN UNION INDICATION}

Omalizumab is indicated in the European Union (EU) as add-on therapy to improve asthma control in adult and adolescent patients $(\geqslant 12$ yrs of age) with severe persistent allergic asthma who have: a positive skin test or in vitro reactivity to a perennial aeroallergen; reduced lung function (forced expiratory volume in one second $<80 \%$ ); frequent daytime symptoms or night-time awakenings; and multiple documented severe asthma exacerbations despite daily high-dose ICS and LABA. It is stated that omalizumab treatment should only be considered for patients with convincing immunoglobulin (Ig)E-mediated asthma (table 1). Omalizumab is indicated for patients with baseline total $\operatorname{IgE}$ levels of $30-700 \mathrm{IU} \cdot \mathrm{mL}^{-1}$. Patients who receive omalizumab should be assessed by their physician after 16 weeks and treatment continued only if there has been a marked improvement in asthma control (table 1).

In order to maximise clinical benefit and cost effectiveness of omalizumab therapy and to minimise inappropriate prescribing, physicians should ensure that patients who are selected for treatment fall within the EU indication [5]. Correct diagnosis is essential and current therapy
CORRESPONDENCE

K-M. Beeh

insaf Respiratory Research Institute

Biebricher Allee 34

D-65187 Wiesbaden

Germany

Fax: 496119854348

E-mail: k.beeh@insaf-wi.de

STATEMENT OF INTEREST

K-M. Beeh has received

compensation for serving on an advisory board and speaking at a conference symposium sponsored by Novartis Pharma $A G$, has participated as a speaker at scientific meetings or courses financed by various companies (Boehringer Ingelheim, Novartis Pharma AG, Pfizer, Fujisawa and Merck, Sharp and Dohme). The institution where K-M. Beeh is currently employed has received compensation for participating in multicentre clinical trials from several companies (AstraZeneca, Boehringer Ingelheim, Novartis Pharma AG, GlaxoSmithKline, Revotar Biopharmaceuticals, EpiGenesis, Pfizer, Corus Pharma, Alimrall Prodesfarma, Merck, Sharp and Dohme and Fujisawa). K-M. Beeh has received travel expenses from Novartis Pharma AG, Boehringer Ingelheim, Merck, Sharp and Dohme and Pfizer for attending several conferences, and has also received funds for research and a fee for organising education. This issue of the European Respiratory Review contains proceedings of a satellite symposium held at the 16th ERS Annual Congress, 2006, which was sponsored by Novartis Pharma AG. The authors were assisted in the preparation of the text by professional medical writers at ACUMED ${ }_{\mathbb{1}}$; this support was funded by Novartis Pharma AG. 


\section{TABLE 1 Using omalizumab in clinical practice}

Therapeutic indication (EU)

Omalizumab is indicated as add-on therapy to improve asthma control in adult and adolescent patients ( $\geqslant 12 \mathrm{yrs}$ of age) with severe persistent allergic asthma who have the following characteristics:

A positive skin test or in vitro reactivity to a perennial allergen

Reduced lung function (FEV1 <80\%)

Frequent daytime symptoms or night-time awakenings

Multiple documented severe asthma exacerbations

Receiving daily high-dose ICS and a LABA

Omalizumab treatment should only be considered for patients with convincing IgE-mediated asthma

Treatment should be initiated by physicians experienced in the diagnosis and treatment of severe persistent asthma

\section{Evaluating response}

16 weeks after commencing omalizumab therapy, patients should be assessed by their physician for treatment effectiveness before further injections are administered. The decision to continue omalizumab therapy should be based on whether a marked improvement in overall asthma control is achieved

EU: European Union; FEV1: forced expiratory volume in one second; ICS: inhaled corticosteroids; LABA: long-acting $\beta_{2}$-agonist; Ig: immunoglobulin.

should be optimised. Patient history, which includes symptoms and spirometry data, should be examined.

\section{DOSING AND ADMINISTRATION}

Omalizumab is administered by subcutaneous injection in the deltoid region of the arm or the thigh every 2 or 4 weeks. Accurate determination of IgE levels, as set out in the dosing table (fig. 1), is a key component of patient selection and correct dosing. Dosage is determined by total serum IgE levels measured before treatment initiation and the patient's body weight. Individual patient dosing requirements are determined using the dosing table.

Omalizumab is indicated for patients with baseline total $\operatorname{IgE}$ 30-700 IU $\cdot \mathrm{mL}^{-1}$. Once baseline serum IgE levels are obtained, there is no need to re-test $\operatorname{IgE}$ levels during omalizumab treatment, because total $\operatorname{IgE}$ levels are elevated during treatment and remain elevated. This phenomenon may appear paradoxical at first glance, but it simply reflects the fact that commercially available $\operatorname{IgE}$ assays also measure complexed
$\operatorname{IgE}$, i.e. $\operatorname{IgE}$ bound to omalizumab. Since these complexes have a longer half-life than free $\mathrm{IgE}$, quantification of $\mathrm{IgE}$ after initiation of omalizumab therapy with conventional assays will result in the detection of higher IgE levels. However, it should be noted that free $\operatorname{IgE}$ decreases rapidly and dramatically during omalizumab treatment.

Significant change in a patient's body weight may necessitate dosing adjustment. Physicians should use the patient's pretreatment serum IgE levels, the new body weight and the dosing table to determine the new dosing regimen. As there is limited experience with self-administration, omalizumab is intended to be administered by a healthcare provider.

\section{CASE STUDY \\ Patient history}

The patient was a 32-yr-old female with childhood-onset asthma, and comorbid allergic rhinitis and atopic dermatitis. She suffered from multiple allergies, including pollen, dust mites, moulds, dog, cat and horse, as confirmed by skin-prick

Total doses mg per 28 days

\begin{tabular}{|c|c|c|c|c|c|c|c|c|c|c|c|}
\hline \multirow{2}{*}{$\begin{array}{l}\text { Baseline } \\
\text { IgE-IU mL }{ }^{-1}\end{array}$} & \multicolumn{10}{|c|}{ Body weight kg } & \\
\hline & $20-25$ & $>25-30$ & $>30-40$ & $>40-50$ & $>50-60$ & $>60-70$ & $>70-80$ & $>80-90$ & $>90-125$ & $>125-150$ & \\
\hline$\geq 30-100$ & 75 & 75 & 75 & 150 & 150 & 150 & 150 & 150 & 300 & 300 & Q4 weeks \\
\hline$>$ 100-200 & 150 & 150 & 150 & 300 & 300 & 300 & 300 & 300 & 450 & 600 & Q2 weeks \\
\hline$>200-300$ & 150 & 150 & 225 & 300 & 300 & 450 & 450 & 450 & 600 & 750 & \\
\hline$>300-400$ & 225 & 225 & 300 & 450 & 450 & 450 & 600 & 600 & & & \\
\hline$>400-500$ & 225 & 300 & 450 & 450 & 600 & 600 & 750 & 750 & & & \\
\hline$>500-600$ & 300 & 300 & 450 & 600 & 600 & 750 & & Do net ar & ninister & & \\
\hline$>600-700$ & 300 & 450 & 450 & 600 & 750 & & & & & & \\
\hline
\end{tabular}

FIGURE 1. Omalizumab dosing table (European Union). Ig: immunoglobulin; Q4 weeks: total dose to be administered every 4 weeks; Q2 weeks: half of total dose to be administered every 2 weeks. 


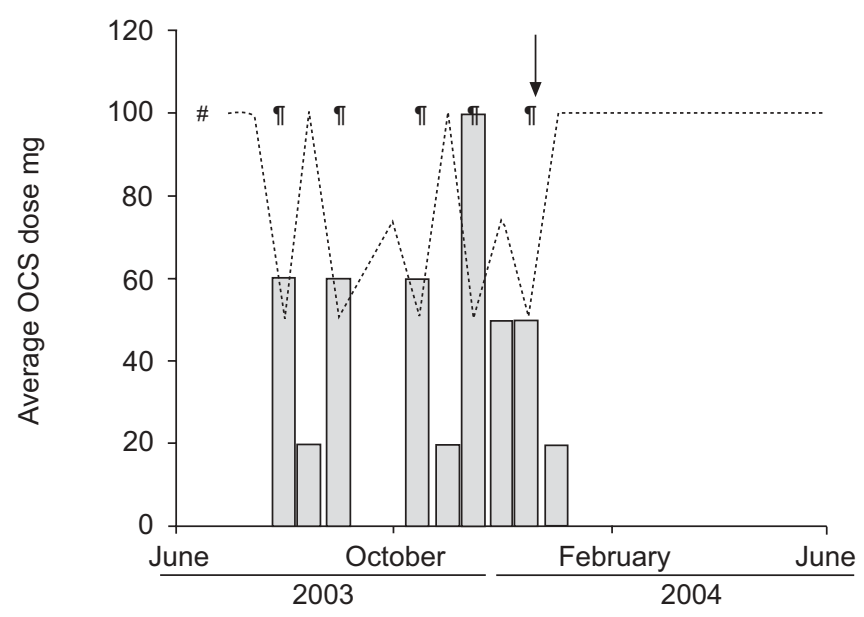

FIGURE 2. Clinical status (….....) and oral corticosteroid (OCS; 1 ) dose of the case-study patient over a period of time. The arrow indicates the start of omalizumab off-label treatment. *: end of the Investigation of Omalizumab in Severe Asthma Treatment (INNOVATE) trial; ${ }^{\imath}$ : time-point at which exacerbations occurred.

tests and radioallergosorbent test classes $\geqslant 2$ for respective allergens. In the 6 months prior to omalizumab initiation, the patient had suffered six exacerbations requiring systemic steroid bursts and had regular night-time symptoms, despite maximal asthma treatment. She had kept a domestic cat until 2001, which was re-homed on physician's advice after a severe exacerbation requiring hospitalisation. However, the patient remained symptomatic at home, due to cat allergen persisting in the environment (fig. 2).

\section{Medication}

Prior to treatment with omalizumab, the patient had been prescribed inhaled fixed combination budesonide/formoterol $320 / 9 \mu \mathrm{g}$, two puffs b.i.d., inhaled fenoterol $100 \mu \mathrm{g}$ p.r.n. $\left(\leqslant 10\right.$ puffs $\cdot$ day $\left.^{-1}\right)$, oral montelukast $10 \mathrm{mg}$ q.d. and an oral $\beta_{2}$-agonist (bambuterol) $20 \mathrm{mg}$ q.d.. She also took a liquid formulation of theophylline on an as-needed basis.

\section{Omalizumab treatment}

Despite high-dose ICS in combination with a LABA, and additional medications, the patient's symptoms remained uncontrolled, and due to frequent exacerbations requiring steroid bursts, it was decided to commence treatment with omalizumab (pre-license era). Based on her body weight of
$60 \mathrm{~kg}$ and a total serum IgE level of $564 \mathrm{IU} \cdot \mathrm{mL}^{-1}$, her dosage of omalizumab was determined to be $300 \mathrm{mg}$ subcutaneously every 2 weeks.

The patient first noticed an improvement in symptoms $\sim 4$ weeks later when collecting her daughter from horse riding. Previous advice from her physician had been to send someone else to collect her daughter in order to avoid asthma exacerbations due to horse allergen, but now she found she "could enter the stables". While she still has severe asthma requiring a high level of medication, she had no severe exacerbations in the following year, demonstrating a clear clinical benefit.

\section{CONCLUSIONS}

Add-on omalizumab is suitable for patients with severe persistent inadequately controlled allergic asthma despite optimised therapy with inhaled corticosteroids and long-acting $\beta_{2}$-agonist, with a history of exacerbations, impaired quality of life and immunoglobulin E levels suitable for dosing. Real-life clinical experience supports the findings of the omalizumab clinical trial programme (see Case study), and shows that add-on omalizumab is clinically effective in reducing symptoms and improving quality of life.

\section{REFERENCES}

1 Humbert M, Beasley R, Ayres J, et al. Benefits of omalizumab as add-on therapy in patients with severe persistent asthma who are inadequately controlled despite best available therapy (GINA 2002 step 4 treatment): INNOVATE. Allergy 2005; 60: 309-316.

2 Tough SC, Hessel PA, Ruff M, Green FH, Mitchell I, Butt JC. Features that distinguish those who die from asthma from community controls with asthma. J Asthma 1998; 35: 657-665.

3 Proceedings of the ATS workshop on refractory asthma: current understanding, recommendations, and unanswered questions. American Thoracic Society. Am J Respir Crit Care Med 2000; 162: 2341-2351.

4 Global Initiative for Asthma. Global strategy for asthma management and prevention. NIH Publication No 02-3659. Bethesda, National Institutes of Health/National Heart, Lung, and Blood Institute, 2005.

5 Brown R, Turk F, Dale P, Bousquet J. Cost-effectiveness of omalizumab in patients with severe persistent allergic asthma. Allergy 2007; 62: 149-153. 